Chimia 46 (1992) 78

(C) Schweiz. Chemiker-Verband; ISSN 0009-429.

\title{
Neue Synthese für Tellurocarbonyl-difluorid
}

\author{
Alois Haas* und Christian Limberg
}

Abstract. Reaction between $\mathrm{CF}_{3} \mathrm{TeTeCF}_{3}$ and $\left(\mathrm{CH}_{3}\right)_{3} \mathrm{SnH}$ in $\mathrm{Et}_{2} \mathrm{O}$ provides $\left(\mathrm{CH}_{3}\right)_{3} \mathrm{SnTeCF}_{3}$ in $75 \%$ yield. This is a good starting material for the preparation of $\mathrm{TeCF}_{2}$ and its cyclic dimer, because pyrolysis of $\left(\mathrm{CH}_{3}\right)_{3} \mathrm{SnTeCF}_{3}$ at $280^{\circ}\left(10^{-3} \mathrm{Torr}\right)$ yields $\mathrm{TeCF}_{2}$ in $60 \%$ yield. On warming from $-196^{\circ}$ to $20^{\circ}, \mathrm{TeCF}_{2}$ dimerizes quantitatively to the corresponding tetrafluoro-1,3-ditelluretane.

Die erst kürzlich berichtete Synthese [1] des ersten, isolierbaren Telluroketons - Tellurocarbonyl-difluorid - hergestellt aus $\mathrm{Hg}\left(\mathrm{TeCF}_{3}\right)_{2}$ und $\left(\mathrm{C}_{2} \mathrm{H}_{5}\right)_{2} \mathrm{Al}(\mathrm{I})$ erfolgte in Analogie zur Synthese von Perfluororganoseleno-ketonen [2]. Die erzielten Ausbeuten waren $\mathrm{ca}$. $10 \%$ und die zugänglichen Substanzmengen infolge einer nur begrenzt einsetzbaren Menge an $\mathrm{Hg}\left(\mathrm{TeCF}_{3}\right)_{2}$ zwangsläufig begrenzt. Nachfolgend wird die Gewinnung von $\mathrm{TeCF}_{2}$ in guten Ausbeuten und präparativen Mengen beschrieben. Sie stützt sich auf ein Verfahren, das ebenfalls zur Synthese von Seleno-ketonen dient, nämlich die Pyrolyse von Verbindungen des Typs $\left(\mathrm{CH}_{3}\right)_{3} \mathrm{SnSeR}_{\mathrm{f}}[3]$. Das analoge Stannyltellan wird durch Umsetzung von $\mathrm{CF}_{3} \mathrm{TeTeCF}_{3}$ mit $\left(\mathrm{CH}_{3}\right)_{3} \mathrm{SnH}$ erhalten. Das sich hierbei primär bildende $\mathrm{CF}_{3} \mathrm{TeH}$ zerfällt in die Ausgangsverbindung $\left(\mathrm{CF}_{3}\right)_{2} \mathrm{Te}_{2}$ und $\mathrm{H}_{2}$, so dass nachfolgende Gesamtreaktion formuliert werden muss:

$\mathrm{CF}_{3} \mathrm{TeTeCF}_{3}+2\left(\mathrm{CH}_{3}\right)_{3} \mathrm{SnH} \rightarrow$ $2\left(\mathrm{CH}_{3}\right)_{3} \mathrm{SnTeCF}_{3}+\mathrm{H}_{2}$

Wird Bis(trifluoromethyl)ditellur mit $\left(\mathrm{CH}_{3}\right)_{3} \mathrm{SnH}$ im Mol-verhältnis 1:2 in $\mathrm{Et}_{2} \mathrm{O}$ umgesetzt, so kann nach Auftrennung des

\footnotetext{
*Korrespondenz: Prof. Dr. A. Haas Lehrstuhl für Anorganische Chemie Il Ruhr-Universität Bochum Postfach 102148

D-4630 Bochum I
}

Reaktionsgemisches durch fraktionierte Kondensation i.V. $\left(\mathrm{CH}_{3}\right)_{3} \mathrm{SnTeCF}_{3}$ in $75 \%$ Ausbeute isoliert werden. Die gelbgefärbte Flüssigkeit ist vollständig charakterisiert worden (s. Experimentelles). Wird nun $\left(\mathrm{CH}_{3}\right)_{3} \mathrm{SnTeCF}_{3}$ bei $280^{\circ}\left(10^{-3}\right.$ Torr) durch ein mit Glaswolle gefülltes Quarzrohr geleitet, so entsteht gemäss

$\left(\mathrm{CH}_{3}\right)_{3} \mathrm{SnTeCF}_{3} \rightarrow\left(\mathrm{CH}_{3}\right)_{3} \mathrm{SnF}+\mathrm{Te}=\mathrm{CF}_{2}$

Tellurocarbonyl-difluorid als tiefviolett gefärbte Substanz, die in einer mit flüssigem $\mathrm{N}_{2}$ gekühlten Falle in 50-60\% Ausbeute kondensiert. In einem vorgeschalteten URohr (auf $-45^{\circ}$ gekühlt) werden $\left(\mathrm{CH}_{3}\right)_{3} \mathrm{SnF}$ und nicht pyrolysiertes Edukt zurückgehalten. Das so gewonnene $\mathrm{TeCF}_{2}$ ist ausreichend rein und steht für weitere Umsetzungen in der auf $-196^{\circ}$ gekühlten Falle zur Verfügung. Nach dem Entfernen der Kühlung wandelt sich das violette Glas augenblicklich in einen roten Feststoff um. Die physikalischen und spektroskopischen Daten des Produktes stimmen mit denen des 2,2,4,4-Tetrafluoro-1,3-ditelluretans [1] vollständig überein.

\section{Experimentelles}

IR-Spektren: BrukerFT-SpektometerIFS 85 ${ }^{13} \mathrm{C}-{ }^{119} \mathrm{Sn}-{ }^{125} \mathrm{Te}-,{ }^{1} \mathrm{H}$ - und ${ }^{19} \mathrm{~F}-\mathrm{NMR}-\mathrm{Spektren}$ Bruker WM 250; $\mathrm{CFCl}_{3}$, TMS, $\left(\mathrm{CH}_{3}\right)_{2} \mathrm{Te}$ und $\left(\mathrm{CH}_{3}\right)_{4} \mathrm{Sn}$ als externe Standards; Locksubstanz und Lsgm.: $\mathrm{CDCl}_{3}$. Massenspektren: Varian MAT CH5 $(70 \mathrm{eV})$; alle tellur- und zinnhaltigen Fragmente zeigen die theoretische Isotopenvertei- lung. Elementaranalysen: Mikroanalytisches Labor der Ruhr-Universität Bochum.

(Trimethylstannyl)(trifluoromethyl)rellan In einem Carius-Rohr mit Teflon-Ventil werden $3,81 \mathrm{~g}(9,7 \mathrm{mmol}) \mathrm{Te}_{2}\left(\mathrm{CF}_{3}\right)_{2}$, gelöst in $5 \mathrm{ml}$ $\mathrm{Et}_{2} \mathrm{O}$, vorgelegt. Anschliessend werden $3,18 \mathrm{~g}$ (19,3 mmol) $\left(\mathrm{CH}_{3}\right)_{3} \mathrm{SnH}$ bei- $196^{\circ}$ hinzukondensiert und danach das Gefäss unter Schütteln auf $20^{\circ}$ erwärmt. Hierbei schlägt die Farbe der vorher orange gefärbten Lsg. nach hellgelb um. Das erhaltene Gemisch wird sofort an einer Stock' schen Vakuumapparatur durch fraktionierte Kondensation aufgetrennt (Fallentemp.: $0^{\circ},-50^{\circ}$ und $\left.-196^{\circ}\right)$. In der auf $-50^{\circ}$ gekühlten Falle werden $5,21 \mathrm{~g}(14,5 \mathrm{mmol})$ reines $\left(\mathrm{CH}_{3}\right)_{3} \mathrm{SnTeCF}_{3}(75 \%)$ erhalten. Das schwach gelb gefärbte Produkt ist luftempfindlich, schmilzt unterhalb $-80^{\circ}$ und ist bei tiefer Temperatur unbegrenzt haltbar. $\mathrm{C}, \mathrm{H}$ Analysen ergaben korrekte Werte. JR (Film zwischen KBr-Platten): 2990w, 2981w, 1374w, $1285 \mathrm{~m}, 1072 v s, 780 s, 722 s, 535 s, 509 m .{ }^{19} \mathrm{~F}-$ NMR: $-10,0(s)$. 'H-NMR: 0,76 (s). ${ }^{13} \mathrm{C}-\mathrm{NMR}$ $-4,0\left(q,{ }^{1} J(\mathrm{C}, \mathrm{H})=132,9, \mathrm{CH}_{3}\right) ; 93,5(q, 1 J(\mathrm{C}, \mathrm{F})=$ $\left.351,3 \mathrm{CF}_{3}\right) .{ }^{125} \mathrm{Te}-\mathrm{NMR}: \delta=248(q),{ }^{2} /\left({ }^{125} \mathrm{Te}, \mathrm{F}\right)$ $=134,3,1 /\left({ }^{125} \mathrm{Te},{ }^{119} \mathrm{Sn}\right)=2215,6,1 /\left({ }^{125} \mathrm{Te},{ }^{117} \mathrm{Sn}\right)=$ 2111,8 , ermittelt durch die Zinn-Sateliten im ${ }^{125}$ Te-NMR. ${ }^{19} \mathrm{Sn}-\mathrm{NMR}: 13.3\left(\mathrm{dec},{ }^{2} /\left({ }^{119} \mathrm{Sn}, \mathrm{H}\right)=\right.$ 54,9). MS: $362\left(25, M^{+}\right), 347\left(35,\left[M-\mathrm{CH}_{3}\right]^{+}\right)$, $317,\left(17, \mathrm{CF}_{3} \mathrm{TeSn}^{+}\right), 261\left(22, \mathrm{CHSnTe}^{+}\right), 248$ $\left(28, \mathrm{SnTe}^{+}\right), 185\left(8,\left(\mathrm{CH}_{3}\right)_{3} \mathrm{SnHF}\right), 169(80$, $\left.\left(\mathrm{CH}_{3}\right)_{2} \mathrm{SnF}\right), 150\left(24,\left(\mathrm{CH}_{3}\right)_{2} \mathrm{Sn}^{+}\right), 135(52$, $\left(\mathrm{CH}_{3} \mathrm{Sn}^{+}\right), 120\left(40, \mathrm{Sn}^{+}\right), 69\left(43, \mathrm{CF}_{3}^{+}\right)$.

Tellurocarbonyl-difluorid und 2,2,4,4-Tetrafluoro-1,3-ditelluretan

Durch ein mit Glaswolle gefülltes Quarzrohr (Länge: $30 \mathrm{~cm}, d=2,5 \mathrm{~cm}$ ) an dessen Ende ein auf $-45^{\circ}$ gekühltes U-Rohr und danach eine mit flüssigem $\mathrm{N}_{2}$ gekühlte Falle angeschlossen sind, werden $0,5 \mathrm{~g}(1,4 \mathrm{mmol})\left(\mathrm{CH}_{3}\right)_{3} \mathrm{SnTeCF}_{3}$ bei $280^{\circ} / 10^{-3}$ Torr geleitet. Die Pyrolysendauer beträgt $c a .0,5 \mathrm{~h}$. In dem auf $-45^{\circ}$ gekühlten U-Rohr werden sowohl $\left(\mathrm{CH}_{3}\right)_{3} \mathrm{SnF}$ als auch das Edukt zurückgehalten (letzteres kann erneut pyrolysiert werden). Der Pegelstand des flüssigen $\mathrm{N}_{2}$ muss kontinuierlich erhöht werden, damit frisch kondensierendes $\mathrm{TeCF}_{2}$ sofort bei- $196^{\circ}$ ausgefroren werden kann, denn nur so kann vermieden werden, dass das extrem temperaturempfindliche $\mathrm{TeCF}_{2}$ bereits beim Kondensieren dimerisiert. Die gesamte innere Fallenwand wird mit einem tiefvioletten Film von $\mathrm{TeCF}_{2}$ überzogen. Nach dem Entfernen der Kühlung tritt sofort Farbänderung nach rot unter Bildung des cyclischen Dimeren auf. Dieses kann problemlos durch fraktionierte Kondensation i.V. gereinigt werden. In einer auf $0^{\circ}$ gekühlten Falle werden 0,15 $(60 \%$, $0,4 \mathrm{mmol}) 2,2,4,4$-Tetrafluor-1,3-ditelluretan isoliert.

Eingegangen am 20. November 1991

[1] R. Boese, A. Haas, C. Limberg, J. Chem. Soc., Chem. Commun. 1991, 1378.

[2] a) A. Darmadi, A. Haas, B. Koch, Z. Naturforsch., B 1980, 35, 526; b) F. Fockenberg, A. Haas, ibid. 1986, 4l, 413; c) R. Boese, A. Haas, M. Spehr, Chem. Ber. 1991, I24, 51.

[3] J. Grobe, D. Le Van, J. Welzel, J. Organomet. Chem. 1990, 386, 321. 